\title{
Cuidado Farmacêutico: Revisão da Farmacoterapia de Usuários da Atenção Primária à Saúde
}

\author{
Pharmaceutical Care: Review of Pharmacotherapy for Primary Care \\ Health Users
}

Recebido em: 10/09/2020 Aceito em: 01/04/2021
Samantha Mayara de Sousa SILVA; Clarissa Oliveira Lima SILVA; Livia Beatriz Nunes de FARIAS; Palloma de Araujo SILVA; Helena Kelly Santos FERREIRA; Bianca Pontes da SILVA; Yasmin Henrique PESSOA; Larissa Santos ARRUDA; Alyne da Silva PORTELA; Romulo Moreira dos SANTOS; Adriana Amorim de Farias LEAL Centro Universitário UNIFACISA, Campus Itararé. Avenida Senador Argemiro de Figueiredo, 1901, Itararé, CEP 58411-020. Campina Grande, PB, Brasil.

E-mail: samanthamayaras@gmail.com

\section{ABSTRACT}

Pharmaceutical services involve clinical assistance activities, mainly focused on health care, and should be committed to direct user support and health outcomes, especially those related to pharmacotherapy. In this context, the study aimed to review the pharmacotherapy of users of Primary Health Care (PHC). A crosssectional study was carried out with a quantitative and descriptive approach. The study was developed from September 2018 to February 2019 at the Basic Health Units (UBS) of Campina Grande-PB, Brazil. The study variables, collected through a structured questionnaire, were classified into demographic and socioeconomic, clinical, and variables related to pharmacotherapy. Of the users observed, the average age was 50 years. In the evaluation of pharmacotherapy, it was observed that the classes of drugs most used were antihypertensive and hypoglycemic due to the morbidity profile related to chronic diseases. There was a $19.8 \%$ frequency of drug interactions among users, some of them being of severe intensity, revealing insecurity of pharmacotherapy, despite the high adherence when questioned about the use. Despite these limitations, the research presented important contributions to the service and health professionals, such as the integration of the pharmacist professional to the family health care teams in the primary care, and the possibility of offering the pharmacotherapy review service to the users, observing the dimensions of need, adherence, effectiveness and safety of the medicines in use

Keywords: pharmaceutical clinical services; pharmacotherapy review; pharmaceutical care.

\section{RESUMO}

Os serviços farmacêuticos envolvem atividades clínico-assistenciais, sobretudo voltadas ao cuidado em saúde, devendo comprometer-se com o apoio direto ao usuário e com a obtenção de resultados em saúde, especialmente os relacionados à farmacoterapia. Nesse contexto, o objetivo do estudo foi revisar a farmacoterapia dos usuários da Atenção Primária à Saúde (APS). Foi realizado um estudo seccional, com abordagem quantitativa e descritiva dos dados. O estudo foi desenvolvido no período de setembro de 2018 
a fevereiro de 2019, nas Unidades Básicas de Saúde (UBS) do município de Campina Grande, PB, Brasil. As variáveis do estudo foram classificadas em demográficas e socioeconômicas, clínicas e relacionadas à farmacoterapia, as quais foram coletadas por meio de um questionário estruturado. Dos usuários observados, a média de idade foi de 50 anos. Na avaliação da farmacoterapia, as classes de medicamentos mais utilizadas foram dos anti-hipertensivos e hipoglicemiantes, devido ao perfil de morbidade relacionada às doenças crônicas. Houve uma frequência de 19,8\% de ocorrência de interações medicamentosas entre os usuários, sendo algumas de intensidade grave, revelando insegurança da farmacoterapia, apesar da elevada adesão quando questionados sobre o uso. Apesar dessas limitações, a pesquisa apresentou contribuições importantes para o serviço e aos profissionais de saúde, como a integração do profissional farmacêutico às equipes de saúde da família na atenção básica, e a possibilidade de oferta do serviço de revisão da farmacoterapia aos usuários, observando as dimensões de necessidade, adesão, efetividade e segurança dos medicamentos em uso.

Palavras-chave: serviços clínicos farmacêuticos; revisão da farmacoterapia; cuidado farmacêutico.

\section{INTRODUÇÃO}

No Brasil, a Atenção Primária à Saúde (APS) apresenta como características primordiais: ser porta de entrada preferencial dos usuários no sistema de saúde; prestar atenção de forma longitudinal e orientada para as pessoas e não focada nos agravos; desenvolver cuidados abrangentes e resolutivos para a maior parte dos problemas de saúde; e coordenar os cuidados quando os usuários necessitarem de atendimento em outros pontos na Rede de Atenção à Saúde (RAS) (1).

No contexto da atenção básica, a Organização Mundial da Saúde (OMS) reconhece a importância do farmacêutico em uma equipe multidisciplinar e interdisciplinar de saúde na prevenção de doenças e na promoção da saúde, pois cabe a esse profissional auxiliar na adesão ao tratamento, incluindo orientação de autovigilância e autocuidado, como medidas preventivas de complicações, seja devido a condição de saúde ou ao uso inadequado de medicamentos, e nas atividades de educação em saúde (2).

Por conseguinte, as atividades da Farmácia Clínica (FC) estão inseridas no contexto da Assistência Farmacêutica. A prática da FC facilita a interação do farmacêutico com o usuário do sistema de saúde, auxiliando no acompanhamento dos pacientes para um efetivo controle da farmacoterapia, otimizando o tratamento farmacológico e prevenindo problemas relacionados com medicamentos (PRM), bem como solucionando problemas que possam surgir durante o processo de uso de medicamentos (3).

Os serviços farmacêuticos envolvem atividades tanto técnico-gerenciais, no sistema de apoio à rede de atenção à saúde, quanto atividades clínico-assistenciais, sobretudo voltadas ao cuidado em saúde, devendo comprometer-se com o apoio direto ao usuário e com a obtenção de resultados em saúde. Dessa forma, no contexto das atividades clínico-assistenciais são desenvolvidos os serviços clínicos farmacêuticos (SCF) (4).

Os serviços farmacêuticos clínicos prestados na atenção primária, por possibilitarem controles sistemáticos das enfermidades crônicas, diminuem o fluxo de atendimentos de usuários portadores destes agravos em unidades de urgência e emergência e, como resultado, minimiza a quantidade de admissões hospitalares. Não obstante, esses mesmos serviços, ao promoverem o uso racional de medicamentos, possibilitam a redução de internações hospitalares associados a eventos adversos a medicamentos (1).

A revisão farmacoterapêutica é um dos SCF regulamentados pelo Conselho Federal de Farmácia (CFF) (5), e se caracteriza como o processo pelo qual as necessidades medicamentosas do usuário são mensuradas a partir da investigação de PRM, de forma organizada, contínua e documentada, com o objetivo de completar os resultados determinados na busca da melhoria da qualidade de vida do usuário. Esse método é geralmente usado para pacientes 
que utilizam simultaneamente vários medicamentos em função de disfunções metabólicos ou por ocorrências de diferentes doenças.

Dessa forma, este trabalho teve como objetivo revisar a farmacoterapia dos usuários de Unidades Básicas de Saúde (UBS) com Estratégia Saúde da Família (ESF).

\section{MÉTODO}

Foi realizado um estudo seccional, com abordagem quantitativa e análise descritiva dos dados. O estudo foi desenvolvido no período de setembro de 2018 a fevereiro de 2019, nas Unidades Básicas de Saúde (UBS) com Estratégia de Saúde da Família (ESF) no município de Campina Grande, $\mathrm{PB}$, vinculadas aos estágios de Saúde Coletiva do Centro Universitário Unifacisa.

Quadro 1. Relação das Unidades Básicas de Saúde (UBS) com Estratégia de Saúde da Família (ESF), por Distrito Sanitário, onde foram coletados os dados da pesquisa, no município de Campina Grande, PB (setembro de 2018 a fevereiro de 2019).

\begin{tabular}{|l|c|}
\hline \multicolumn{1}{|c|}{ UBS } & $\begin{array}{c}\text { Distrito } \\
\text { Sanitário }\end{array}$ \\
\hline Campos Sales & I \\
\hline Francisco Brasileiro - Equipes I e II & I \\
\hline Malvinas II & VI \\
\hline Pedreira & IV \\
\hline Raiff Ramalho- Equipes I e II & IX \\
\hline Ricardo Amorim & X \\
\hline Ronaldo Cunha Lima - Equipes I e II & V \\
\hline Severino de Souza Costa & IX \\
\hline Tambor I & IV \\
\hline Tambor II & IV \\
\hline
\end{tabular}

Secretaria Municipal de Saúde, 2019 (6).

No período da execução da pesquisa, a APS do município era composta por 107 equipes de saúde da família (eSF), organizadas em 10 Distritos Sanitários, e, no momento da coleta de dados dessa pesquisa, 10 UBS estavam vinculadas ao estágio de saúde coletiva do curso de Farmácia da instituição (Quadro 1), nas quais a pesquisa foi desenvolvida.

Foram considerados como critérios de inclusão, pacientes de ambos os sexos, na idade a partir de 20 anos (adultos e idosos), pacientes que apresentavam prescrição médica com necessidade de revisão, pertencentes à área de abrangência das unidades de saúde pesquisadas pelo projeto, e que possuíam cadastro atualizado na UBS. Os critérios de exclusão foram os pacientes que não apresentavam nenhuma prescrição medicamentosa passível de revisão e que não aceitarem participar da pesquisa e recusarem ou estarem impossibilitados de assinar o termo de consentimento livre e esclarecido (TCLE).

Dessa forma, o processo de amostragem foi por conveniência, a partir dos pacientes oriundos das populações de abrangência das referidas UBS, que procuraram o serviço durante o período da pesquisa e atenderam aos critérios de inclusão, totalizando 91 pacientes.

As variáveis do estudo foram classificadas em variáveis demográficas e socioeconômicas (sexo, idade, anos de estudo e renda), variáveis clínicas (morbidades, antecedentes familiares; alergias; pressão arterial sistólica e diastólica, glicemia capilar, circunferência abdominal e índice de massa corporal para os adultos e variáveis relacionadas à farmacoterapia foi avaliado se o usuário utiliza ou não o medicamento, a quantidade de medicamentos prescritos e não prescritos utilizados, e, dentre os prescritos, foi avaliada a segurança quanto as possíveis interações medicamentosas, entre os medicamentos prescritos e não prescritos utilizados pelos pacientes.

Para a coleta de dados foi utilizado, como instrumento, um formulário estruturado contendo informações referentes às variáveis do estudo, sendo preenchido a partir de entrevista com os pacientes na UBS e complementando as informações, principalmente sobre a utilização dos medicamentos a partir dos prontuários físicos de cada paciente, disponíveis nas UBS.

Quanto à aferição das variáveis clínicas, foram utilizados tensiômetro $\mathrm{BIC}^{\circledR}$, glicosímetro Accu-Chek ${ }^{\circledR}$; balança Digi-Health Serene Multilaser ${ }^{\mathbb{R}}$ com capacidade máxima de 150 quilos e fita métri- 
ca inelástica Vonder $^{\circledR}$ de 1,5 metros. A avaliação do estado nutricional foi realizada a partir do cálculo do IMC (quociente entre o peso, em quilogramas, e o quadrado da estatura, em metros). Os usuários foram pesados, estando sem sapatos, com os pés unidos no centro da balança, corpo ereto de forma que o peso fosse distribuído igualmente nos dois pés. A estatura foi medida no estadiômetro da balança mecânica, com os participantes em pé, descalços, com os braços estendidos ao longo do corpo, cabeça mantida em plano Frankfurt e sem adorno na cabeça. Para a análise do IMC foram utilizados os critérios de diagnóstico nutricional recomendados para a população adulta pela OMS (7) (Quadro 2).

Quadro 2. Classificação do índice de massa corporal para adultos.

\begin{tabular}{|l|c|}
\hline \multicolumn{1}{|c|}{ Classificação } & IMC $\left(\mathrm{Kg} / \mathrm{m}^{2}\right)$ \\
\hline Baixo peso & $\leq 18,5$ \\
\hline Normal & $18,6-24,9$ \\
\hline Sobrepeso & $25-29,9$ \\
\hline Obesidade & $\geq 30$ \\
\hline
\end{tabular}

Organização Mundial de Saúde, 1995.

A circunferência abdominal foi mensurada com fita métrica inelástica com precisão de 0,01 $\mathrm{cm}$ e comprimento de $150 \mathrm{~cm}$. Foram considerados elevados os valores maiores ou iguais a $94 \mathrm{~cm}$ para homens e maiores ou iguais a $80 \mathrm{~cm}$ para as mulheres, de acordo com as Diretrizes Brasileiras de Obesidade (8).

A pressão arterial foi aferida em três momentos por método auscultatório, correspondentes às fases I e V dos sons de Korotkoff, com intervalo de um minuto, realizados pelo mesmo pesquisador, com o usuário sentado e em repouso prévio de pelo menos cinco minutos, utilizando um esfigmomanômetro, sendo considerada a média entre os dois últimos valores. Quando a medida das pressões sistólica e/ ou diastólica apresentaram diferença maior que 4 $\mathrm{mmHg}$, a aferição foi realizada novamente até que se obtivessem medidas com diferença inferior ou igual a esse valor. Os critérios para diagnóstico de pressão arterial elevada seguirão as Diretrizes Brasileiras de Hipertensão (9).
A glicemia capilar casual foi medida por glicosímetro e foi considerado valor elevado na observação do controle glicêmico aqueles maiores iguais a $200 \mathrm{mg} / \mathrm{dL}$, de acordo com as Diretrizes Brasileiras de Diabetes (10).

Para a obtenção destes parâmetros, os pesquisadores foram treinados previamente à coleta de dados para aferição destas medidas, de forma a seguir com os parâmetros dispostos pelas diretrizes.

Em relação a farmacoterapia, os medicamentos utilizados pelos pacientes foram identificados por meio da análise das prescrições trazidas pelos mesmos e questionamento sobre uso de medicamentos não prescritos por meio de uma entrevista. A classificação do risco de interações medicamentosas foi realizada com o auxílio das ferramentas online gratuitas Medscape ${ }^{\circledR}$ (https://www.medscape.com/) e Micromedex Solutions ${ }^{\circledR}$ (https://www. micromedexsolutions.com), e foram classificadas de acordo com a intensidade dos efeitos, a saber: grave, para as interações potencialmente fatais ou capazes de causar danos permanentes; moderado, considerando a interação que provoca a deterioração do paciente, necessitando de tratamento adicional, hospitalização ou aumento da permanência hospitalar; e leve, cujo efeito é geralmente passageiro e pode ser desconfortável ou despercebido, mas que não afetam significativamente o efeito da terapia e não exige tratamento adicional.

Os dados foram organizados em planilha do programa Microsoft Excel 97/2003 e os resultados foram analisados e expressos em distribuição de frequências (absolutas e relativas), apresentação dos valores máximos e mínimos, medidas de tendência central (médias) e de dispersão (desvio padrão), por meio do Statistical Package for the Social Sciences - SPSS, versão 20.0.

$\mathrm{O}$ projeto foi registrado na Plataforma Brasil, avaliado e aprovado pelo Comitê de Ética em Pesquisa da Unifacisa sob CAAE $n^{\circ}$ 94708418.2.0000.5175, considerando a Resolução 466/2012 do Conselho Nacional da Saúde, sobre pesquisa envolvendo seres humanos (11) e aprovado pelo Comitê de Ética em Pesquisa (CEP) CESED. Dessa forma, os pacientes foram informados antecipadamente sobre os objetivos do estudo, sendo-lhes garantidos o direito a desistência, bem 
como o sigilo, salvaguardando o direito da privacidade, não havendo necessidade de identificação do mesmo.

A coleta de dados obedeceu à exigência da assinatura do Termo de Consentimento Livre Esclarecido (TCLE). Da mesma forma, os dados que foram coletados a partir do prontuário do usuário, obedecendo à exigência da assinatura do Termo de autorização para pesquisa em arquivos ou documentos.

\section{RESULTADOS E DISCUSSÃO}

Durante o período do estudo foi revisada a farmacoterapia de 91 pacientes, dos 122 que frequentaram a UBS nos turnos em que os pesquisadores se encontravam para a coleta de dados.

Dos usuários observados, a média de idade foi de 50 (dp 13,6) anos, com idade mínima de 20 e máxima de 80 anos. As variáveis demográficas e socioeconômicas estão apresentadas na Tabela 1.

Tabela 1. Distribuição da frequência das variáveis demográficas e socioeconômicas dos usuários atendidos em Unidade Básica de Saúde, no município de Campina Grande, PB (setembro de 2018 a fevereiro de 2019).

\begin{tabular}{|c|c|c|}
\hline \multicolumn{1}{|c|}{ Variável } & N & $\%$ \\
\hline Sexo & & \\
\hline Feminino & 81 & 89,0 \\
\hline Masculino & 10 & 11,0 \\
\hline Anos de estudo & & \\
\hline Menos de 1 ano & 07 & 7,7 \\
\hline 1 a 7 anos & 36 & 39,6 \\
\hline 8 anos ou mais & 48 & 52,7 \\
\hline Renda & & \\
\hline Menos que 1 salário mínimo & 03 & 3,3 \\
\hline 1 a 2 salários mínimos & 51 & 56,0 \\
\hline 2,1 a 3 salários mínimos & 31 & 34,1 \\
\hline Mais que 3 salários mínimos & 06 & 6,6 \\
\hline TOTAL & 91 & 100,0 \\
\hline
\end{tabular}

De maneira geral, as morbidades mais frequentes presentes na amostra estudada foram Hipertensão Arterial Sistêmica, Diabetes Mellitus tipo 2 e Hipercolesterolemia, com uma média de 2,1 morbidades por paciente. Em relação aos parâmetros clínicos observados, a média de pressão arterial sistólica foi de 130,33 (dp 17,85) $\mathrm{mmHg}$, de pressão arterial diastólica foi de 83,85 (dp 16,65) $\mathrm{mmHg}$, de glicemia capilar foi de $137,24 \mathrm{mg} / \mathrm{dL}$ e de circunferência abdominal foi de $98,50(\mathrm{dp} \mathrm{12,82)} \mathrm{cm}$. A distribuição da frequência dessas variáveis está descrita na Tabela 2.

O índice de massa corporal (IMC) foi calculado apenas para os pacientes adultos, conforme orientação da OMS (7) por não ser um parâmetro adequado para a pessoa idosa. Desta forma o IMC foi calculado para 68 usuários, que correspondeu a $74,7 \%$ dos adultos pesquisados, cuja frequência de classificação está representada na Figura 1.

Tabela 2. Distribuição da frequência dos parâmetros clínicos dos usuários atendidos em Unidade Básica de Saúde, no município de Campina Grande - PB (setembro de 2018 a fevereiro de 2019)

\begin{tabular}{|c|c|c|}
\hline Variável & $\mathbf{N}$ & $\%$ \\
\hline \multicolumn{3}{|l|}{ Pressão arterial sistólica } \\
\hline Normal & 57 & 52,6 \\
\hline Alterada $(\geq 140 \mathrm{mmHg}$ ) & 34 & 37,4 \\
\hline \multicolumn{3}{|l|}{ Pressão arterial diastólica } \\
\hline Normal & 60 & 65,9 \\
\hline Alterada ( $\geq 90 \mathrm{mmHg}$ ) & 31 & 34,1 \\
\hline \multicolumn{3}{|l|}{ Glicemia capilar } \\
\hline Normal & 81 & 89,0 \\
\hline Alterada $(\geq 200 \mathrm{mg} / \mathrm{dL})$ & 10 & 11,0 \\
\hline \multicolumn{3}{|l|}{ Circunferência abdominal } \\
\hline Normal & 19 & 20,9 \\
\hline $\begin{array}{l}\text { Alterada (Homens: } \geq 102 \mathrm{~cm} \text {; } \\
\text { Mulheres: } \geq 88 \mathrm{~cm} \text { ) }\end{array}$ & 58 & 63,7 \\
\hline Não aferido & 14 & 15,4 \\
\hline TOTAL & 91 & 100,0 \\
\hline
\end{tabular}


Figura 1. Distribuição da frequência da classificação do Índice de Massa Corporal dos usuários adultos (20 a 59 anos) atendidos em Unidade Básica de Saúde, no município de Campina Grande, PB (setembro de 2018 a fevereiro de 2019).

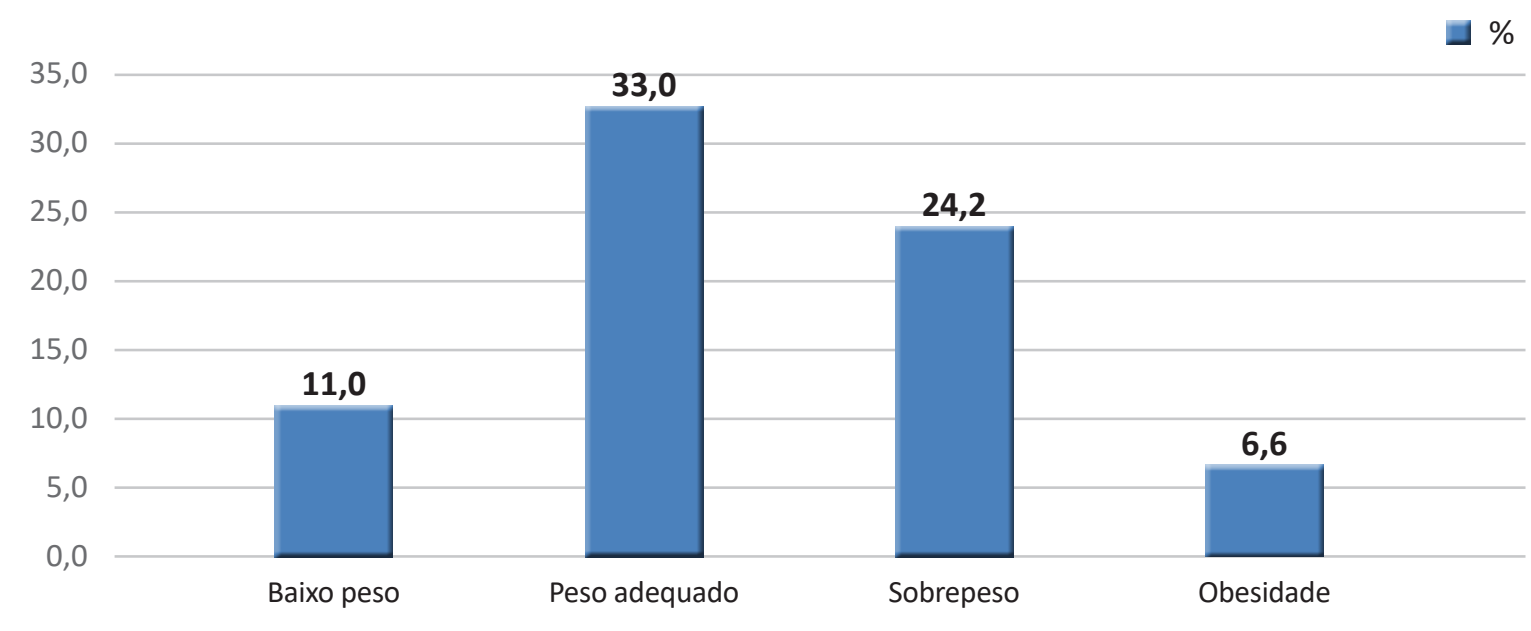

Classificação do Índice de Massa Corporal

Quanto aos dados clínicos também foram avaliados os antecedentes familiares dos pacientes, cuja distribuição de frequência está apresentada na Figura 2. Na avaliação da frequência da farmacoterapia, as classes de medicamentos mais utilizadas foram dos anti-hipertensivos e antidiabéticos, de acordo com as prescrições trazidas pelos pacientes, seguindo o mesmo perfil das morbidades mais frequentes. A distribuição desta frequência está representada na Tabela 3 e a classificação das interações medicamentosas está disposta no Quadro 3. Dentre os medicamentos em uso, foi observado que 18 usuários $(19,8 \%)$ apresentaram ao menos uma interação medicamentosa. As principais interações medicamentosas observadas no estudo estão descritas no Quadro 3.

Figura 2. Distribuição da frequência dos antecedentes familiares dos usuários atendidos em Unidade Básica de Saúde, no município de Campina Grande, PB (setembro de 2018 a fevereiro de 2019).

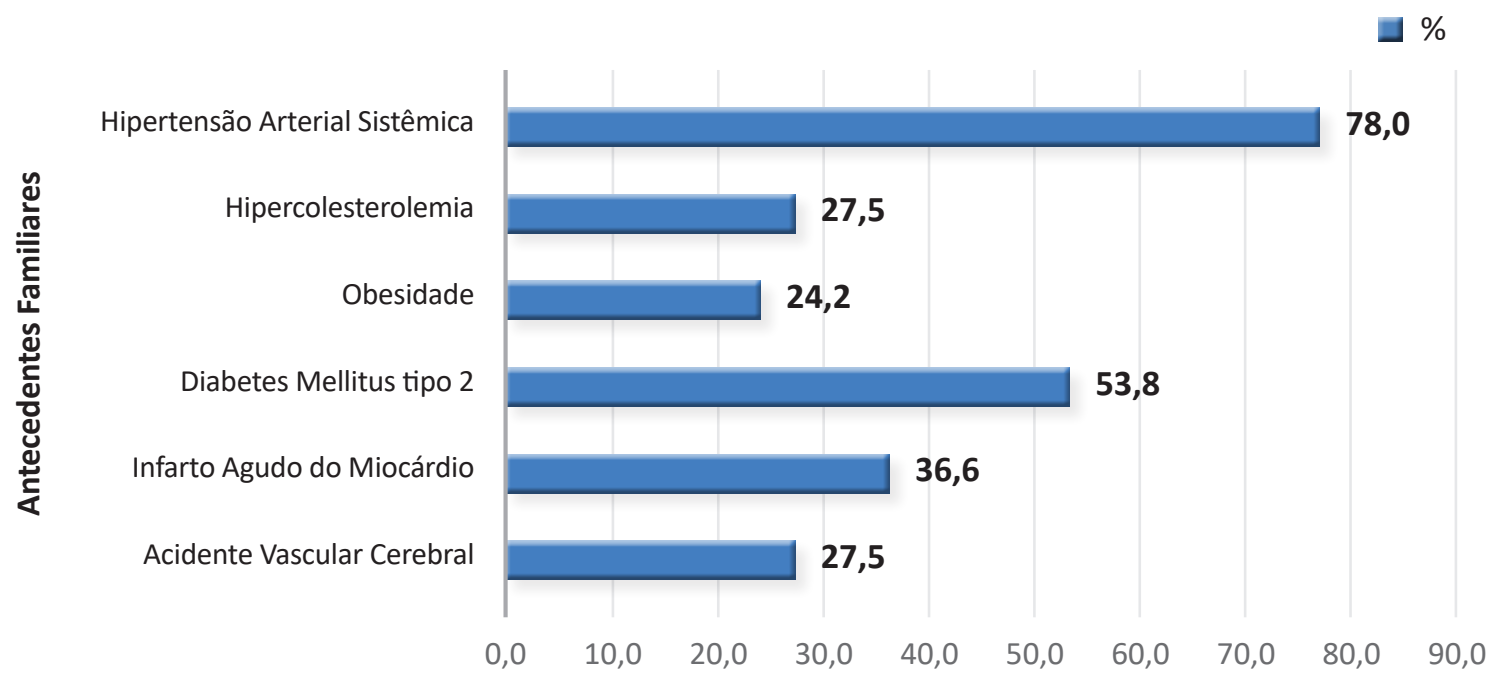


Tabela 3. Distribuição da frequência das variáveis relacionadas à farmacoterapia dos usuários atendidos em Unidade Básica de Saúde, no município de Campina Grande, PB (setembro de 2018 a fevereiro de 2019).

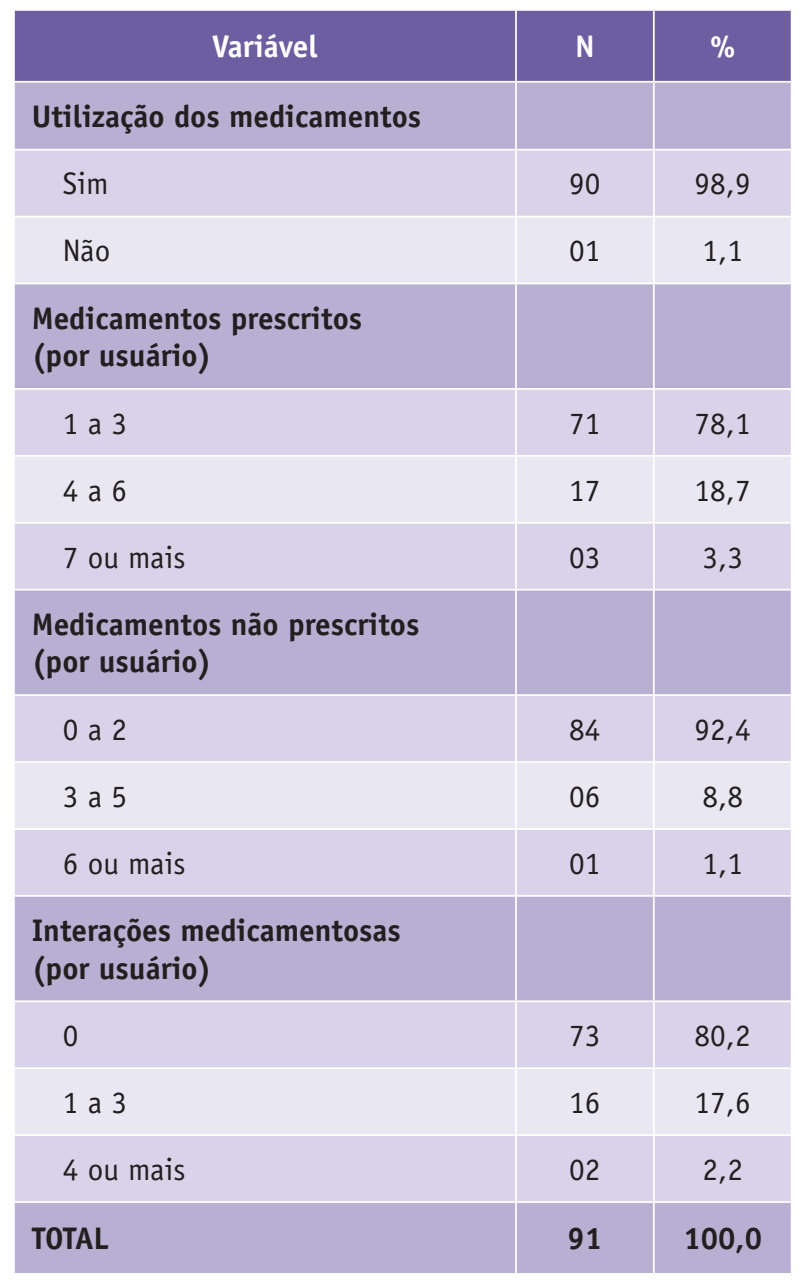

A Atenção Primária à Saúde (APS) vem se desenvolvendo há décadas, recebendo diferentes denominações e abordagens e, com a implantação do Sistema Único de Saúde (SUS) é considerada a ordenadora do cuidado, enfatizando a reorientação do modelo assistencial, a partir de um sistema universal e integrado de atenção à saúde, para a utilização dos serviços ofertados a população em todos os níveis de complexidade (13). Ainda, a APS é destaque na política nacional de saúde, possuindo papel central para que o SUS propicie acesso à atenção à saúde integral e de qualidade à população, incluindo o acesso e a utilização adequada de medicamentos $(14,15)$.
Guibi e cols (16) indicaram que a maioria dos usuários que frequentam a atenção primária são mulheres, com ensino fundamental e renda que as classificam na classe social tipo C, como observado em estudos nacionais e internacionais. Embora os homens possuam maiores taxas de morbimortalidade, eles resistem mais a buscar a assistência primária, um dos motivos da criação, pelo Ministério da Saúde, da Política Nacional de Atenção Integral à Saúde do Homem (17). O fato da proporção de homens com 60 anos ou mais ser bem maior do que a de mulheres desta mesma faixa etária, estar relacionado ao fato no qual os homens só procuram atendimento à saúde quando mais velhos e provavelmente com sintomas (17).

No âmbito da assistência farmacêutica fornecida na APS, são ofertados os serviços clínicos, contextualizados no modelo de prática do Cuidado Farmacêutico, nos diversos pontos de atenção à saúde desse nível de atenção, na tentativa de suprir a carência de padronização de procedimentos e de instrumentos de avaliação de desfechos, sendo esta carência uma das responsáveis pela ineficiência na detecção, prevenção e resolução dos problemas apresentados pelos pacientes em relação ao uso de medicamentos e na mensuração dos desfechos em saúde (18).

Os serviços clínicos responsabilizam o farmacêutico pelo cuidado em saúde e são realizados a partir da disponibilidade para o paciente, do rastreamento em saúde, das ações de prevenção e promoção da saúde, da contribuição para efetividade dos medicamentos, da prevenção ao dano associado ao uso do medicamento, do uso responsável dos limitados recursos de saúde e da promoção da segurança do paciente. Desse modo, os serviços e procedimentos farmacêuticos que podem ser ofertados na APS vão desde a aferição da glicemia capilar até o acompanhamento farmacoterapêutico (19).

Nesse contexto, a revisão da farmacoterapia é um serviço no qual o farmacêutico faz uma análise estruturada e crítica sobre os medicamentos utilizados pelo paciente, com os objetivos de minimizar a ocorrência de problemas relacionados à farmacoterapia, melhorar a adesão ao tratamento e os resultados terapêuticos, bem como reduzir o desperdício de recursos (5). 
Quadro 3. Descrição das potenciais interações medicamentosas, por grau de interação, dos medicamentos utilizados pelos usuários atendidos em Unidade Básica de Saúde, no município de Campina Grande, PB (setembro de 2018 a fevereiro de 2019).

\begin{tabular}{|c|c|c|}
\hline \multirow{2}{*}{ Medicamento } & \multicolumn{2}{|l|}{ Interação } \\
\hline & Descrição & Grau \\
\hline Carbonato de Lítio e Losartana & Aumento do risco de toxicidade por lítio & Grave \\
\hline Citalopram e Quetiapina & Aumento do risco de prolongamento do intervalo QT. & Grave \\
\hline Fenobarbital e Diazepam & Depressão respiratória & Grave \\
\hline Hidroclorotiazida e Ácido Acetilsalicílico & Nefrotoxicidade; Redução da eficácia do Hidroclorotiazida & Grave \\
\hline Hidroclorotiazida e Diclofenaco & Nefrotoxicidade; Redução da eficácia do Hidroclorotiazida & Grave \\
\hline Metformina e Ácido Acetilsalicílico & Aumento do risco de hipoglicemia & Grave \\
\hline Acelato Noretisterona e Amitripilina & Eficácia antidepressiva; toxicidade tricíclica & Leve \\
\hline Atenolol e Carbonato de cálcio & Diminuição da absorção do atenolol & Leve \\
\hline Diazepam e Omeprazol & Aumenta e prolonga o efeito do Diazepam & Leve \\
\hline Atenolol e Ácido Acetilsalicílico & Aumento da pressão arterial & Moderada \\
\hline Atenolol e Metformina & Resulta em hipoglicemia ou hiperglicemia & Moderada \\
\hline Captopril e hidroclorotiazida & Redução da pressão arterial & Moderada \\
\hline Captopril e Metformina & Aumento do risco de hipoglicemia & Moderada \\
\hline Enalapril e Metformina & Aumento do risco de hipoglicemia & Moderada \\
\hline Enalapril e Hidroclorotiazida & Redução da Pressão Arterial & Moderada \\
\hline Losartana e Diclofenaco & Aumento da pressão arterial; Disfunção renal & Moderada \\
\hline Losartana e Ibuprofeno & Disfunção renal ou Aumento da Pressão Arterial & Moderada \\
\hline Metformina e Propranolol & Hiperglicemia & Moderada \\
\hline
\end{tabular}

Micromedex Solutions ${ }^{\circledR}(12)$.

O presente estudo apresenta achados em consonância com Pesquisa Nacional de Utilização de Medicamentos (PANUM), em relação ao o perfil demográfico, social e clínico dos usuários da APS no Brasil, e ao perfil de utilização de medicamentos, no tocante aos usuários com uma ou mais morbidades (20).

Dessa forma, a revisão da farmacoterapia desses usuários, de maneira coletiva, no presente estudo, revelou aspectos comuns ao estudo sobre a PNAUM realizado por Bermudez e cols. (2016), que abordaram que dada a relevância das doenças crônicas no perfil da morbimortalidade brasileira e avaliando o acesso a medicamentos para o tratamento de doenças crônicas não transmissíveis, 94,3\% dos brasileiros com alguma das doenças crônicas pesquisadas pela PNAUM, e que tiveram prescrição de medicamento para o controle da doença, tiveram acesso e utilizaram o medicamento prescrito. Ressalte-se que esse acesso foi total, ou seja, a todos os medicamentos prescritos. Apenas $2,6 \%$ dos indivíduos não haviam usado os medicamentos prescritos nos últimos 30 dias. Os autores avaliaram que as políticas nacionais de medicamentos e de assistência farmacêutica implementadas no Brasil a partir de 1999 demonstram estar atingindo o objetivo de ampliação de acesso e redução de inequidades (21).

É importante essa avaliação realizada pelo profissional farmacêutico, especialmente na atenção básica, pois torna possível prevenir os erros e danos causados ao paciente; a falta de adesão ao tratamento das doenças crônicas não transmissíveis resulta em alto índice de mortalidade (22). 
Entretanto, se houvesse o tratamento correto e consequentemente controle da Hipertensão Arterial e Diabetes Mellitus tipo 2, por exemplo, a redução dos gastos chegaria a cerca de $\mathrm{R} \$ 840$ milhões em custos anuais para o governo, o que minimizaria as complicações e resultaria em maior qualidade de vida para os pacientes $(22,23)$.

Os pacientes avaliados pelo estudo atual apresentaram uma média de uso de 2,1 medicamentos por usuário. $\mathrm{O}$ uso concomitante de medicamentos já é apontado na literatura como um fator de baixa adesão à farmacoterapia e, consequentemente, ao uso desnecessário ou ineficiência do uso, dimensão que é avaliada durante o serviço de revisão da farmacoterapia. Além disso, o uso abusivo de medicamentos, causados pela desinformação dos prescritores, dispensadores e pela automedicação também são fatores que induzem a ineficácia do tratamento e propiciam a ocorrência de Interações Medicamentosas (IM) (23).

Essas situações indicam que medidas devem ser tomadas a fim de melhorar a qualidade dos cuidados no sistema de saúde, como educação continuada para os prescritores e melhoria das condições de dispensa nessas unidades. Desta forma, é importante identificar potenciais interações medicamentosas durante o tratamento das doenças crônicas e realizar manejo farmacoterapêutico adequado para evitar efeitos adversos graves ou até a morte $(23,24)$.

Foram observadas possíveis interações medicamentosas como sinergismos, diminuições e aumento do efeito/absorção e níveis de drogas, intoxicações, e efeitos plasmáticos e intestinais, em diversos graus (leve, moderado e grave), às quais devem ser consideradas importantes, pois poderão causar igual desconforto para o usuário podendo levá-lo a não adesão, assim como no indivíduo que apresenta sintomas mais graves. A participação do farmacêutico e o processo do cuidado farmacêutico podem, portanto, propiciar informação e conhecimento confiável e de alta relevância a população, o que poderia tornar o tratamento farmacológico mais seguro e benéfico (25).
Uma vez inserido em um serviço clínico farmacêutico, existem muitos benefícios relatados pelos pacientes, desde uma melhor compreensão sobre os seus medicamentos e doenças, até a autoconfiança e autocontrole sobre o seu uso. Sendo um cenário propício para construção de uma relação positiva com o paciente, abrindo a possibilidade de compartilhar as decisões terapêuticas, alguns aspectos são importantes, como reconhecer os indivíduos na sua integralidade, promovendo o cuidado centrado no paciente, que são atributos importantes para adesão ao cuidado oferecido pelo farmacêutico (26).

Diante deste cenário, fica clara a importância da atenção farmacêutica na sociedade, visto que o profissional farmacêutico auxilia na diminuição dos índices de automedicação, evitando o uso de doses e medicamentos inadequados. A orientação da população a partir do farmacêutico é fundamental para o uso consciente de medicamentos (27).

\section{CONCLUSÃO}

A pesquisa apresentou algumas limitações que devem ser consideradas. O estudo abordou pacientes de unidades básicas de saúde, portanto outros níveis de atendimento não foram incluídos e apenas alguns dos pacientes foram atendidos durante os turnos da pesquisa, além de, em alguns momentos, ter ocorrido deficiência de oferta de material como a balança mecânica para aferição de peso e altura. Sugere-se cuidado na extrapolação dos dados para populações com características sociodemográficas distintas. Novos estudos de base populacional e prospectivos devem ser realizados para melhor clareza dessa problemática.

Apesar dessas limitações, a pesquisa apresentou contribuições importantes para o serviço e aos profissionais de saúde, como a integração do profissional farmacêutico às equipes de saúde da família na atenção básica, e a possibilidade de oferta do serviço de revisão da farmacoterapia aos usuários, observando as dimensões de necessidade, adesão, efetividade e segurança dos medicamentos em uso. 
1. Barros DSL, Silva DLM, Leite SN. Serviços Farmacêuticos Clínicos da Atenção Primária à Saúde do Distrito Federal. Brasília. Tese [Doutorado em Ciências Farmacêuticas] - Universidade de Brasília, 2018.

2. Gomes KKLB, Brito MRM, Gláucio BS, Rivelilson MF. Atenção farmacêutica a um portador de diabetes: relato de caso. Bol. Inf. Geum. 2014;5(1):108-121.

3. Araújo OS, Costa EA, Guerra Junior AA, Acurcio FA, Guibu IA, Álvares, Costa KS, Karnikowski, MGO, Soeiro OM, Leite SN. Atividades farmacêuticas de natureza clínica na atenção básica no Brasil. Rev. Saúde Pública. 2017; 51 (Suppl 2): 6s. DOI: 10.11606/S15188787.2017051007109.

4. Alcântara AP, Terra Júnior AT. Intervenção Farmacêutica Junto a Atenção Básica de Saúde da Família. Rev. Cient. Educ Meio Amb. 2016;7(2):13-32. DOI: 10.31072/rcf. v7i2.406.

5. CFF. Serviços farmacêuticos diretamente destinados ao paciente, à família e à comunidade: contextualização e arcabouço conceitual / Conselho Federal de Farmácia. Brasília: Conselho Federal de Farmácia, 2016.

6. SMSCG. Estratégia Saúde da Família. Campina Grande. Secretaria Municipal de Saúde de Campina Grande/PB. 2019.

7. Organização Mundial de Saúde - OMS. Physical status: the use and interpretation of anthropometry. Geneva: WHO; 1995.

8. ABESO. Associação Brasileira para o Estudo da Obesidade e da Síndrome Metabólica. Diretrizes brasileiras de obesidade. São Paulo, 2016.

9. SBC. Sociedade Brasileira de Cardiologia. VII Diretrizes brasileiras de hipertensão. Arq Bras Cardiol. 2016;107(3):1-83.

10. SBD. Diretrizes da Sociedade Brasileira de Diabetes: 2017-2018. Sociedade Brasileira de Diabetes. São Paulo: Clannad. 2017.

11. BRASIL. Resolução no 466, de 12 de dezembro de 2012. Ministério da Saúde. Brasília: Diário Oficial União. 2013.

12. Healthcare Series Micromedex, USA, IBM Micromedex Drug Ref. 2019. Disponível em: https://www. micromedexsolutions.com/.

13. Marsiglia, RMG. Universalização do acesso ao Sistema Único de Saúde no Brasil: desafios para a Atenção Primária à Saúde. Cad. Terapia. Ocup. 2012;20(3): 317-325. DOI: 10.4322/cto.2012.032.

14. BRASIL. Aprova a Política Nacional de Atenção Básica, estabelecendo a revisão de diretrizes para a organização da Atenção Básica, no âmbito do Sistema Único de Saúde (SUS). Portaria $n^{\circ} 2.436$, de 21 de setembro de 2017. Ministério da Saúde. Diário Oficial da União. 2017 (38).

15. Schmidt MI, Duncan BB, Silva GA, Menezes AM, Monteiro CA, Barreto SM, Chor D, Menezes PR. Doenças crônicas não transmissíveis no Brasil: carga e desafios atuais. The Lancet. 2011;61-73. DOI: 10.1016/ S0140-6736(11)60135-9.

16. Guibu IA, Moraes JC, Guerra Junior AA, Costa EA, Acurcio FA,Costa KS, Karnikowski MGO, Soeiro OM, Leite SN, Álvares J. Características principais dos usuários dos serviços de atenção primária à saúde no Brasil. Rev. Saúde Pública. 2017;51(Suppl 2): 17s. DOI: 10.11606/S1518-8787.2017051007070.

17. BRASIL. Institui a Portaria $n^{\circ} 1944$, de 28 de agosto de 2009. Ministério da Saúde. Brasília: Diário Oficial da União. 2009.

18. Souza, TT. Desenvolvimento de modelos de serviços de cuidado farmacêutico a pacientes polimedicados. Tese (Doutorado em Ciências Farmacêuticas) - Universidade Federal do Paraná (UFP), Curitiba, 2017.

19. Costa KS, Nascimento Junior JM, Soeiro OM, Paganelli MO, De Araujo SQ. Assistência farmacêutica nas Redes de Atenção à Saúde do SUS. p. 25-36. In: BRASIL. Ministério da Saúde. Secretaria de Ciência, Tecnologia e Insumos Estratégicos. Departamento de Assistência Farmacêutica e Insumos Estratégicos. Cuidado Farmacêutico na Atenção Básica. Caderno 1: Serviços Farmacêuticos na Atenção Básica. Brasília: Ministério da Saúde, 2014.

20. Bertoldi AD, Dal Pizzo TS, Ramos LR, Mengue SS, Luiza VL, Tavares NUL, Farias MR, Oliveira MA, Arrais PSD. Perfil sociodemográfico dos usuários de medicamentos no Brasil: resultados da PNAUM 2014. Rev. Saúde Pública. 2016;50(Suppl 2):5s. DOI: 10.1590/ s1518-8787.2016050006119.

21. Bermudez JAZ, Barros MBA. Perfil do acesso e da utilização de medicamentos da população brasileira - contribuições e desafios da PNAUM - Inquérito Domiciliar. Rev. de Saúde Pública. 2016;50(2):1-4. DOI:10.1590/S1518-8787.201605000SUPL2AP.

22. Kawano DF, Pereira LRL, Ueta JM, Freitas O. Acidentes com os medicamentos: como minimizá-los? Rev. Cienc Farm. 2006;42(4): 487-495.

23. Alves NR, Menezes PDL, Diniz JÁ, Souza FAF, Carvalho PMM, Tavares SMQMS. Avaliação das interações medicamentosas entre antihipertensivos e hipoglicemiantes orais. Rev. Mult. Psicol. 2019;13(44):374-392. DOI: 10.14295/idonline.v13i44.1625. 
24. Araújo SQ, Costa KS, Luiza VL, Lavras C, Santana EA, Tavares NUL. Organização dos serviços farmacêuticos no Sistema Único de Saúde em regiões de saúde. Ciênc. Saúde Colet. 2017;22(4):1181-1191. DOI: 10.1590/141381232017224.27042016 .

25. Iamaguchi FE, Crozatti MTL, Bertolini DA, Demarchi IG, Okawa RTP, Teixeira JJV. Frequência de Interações Potenciais Medicamento-Medicamento em Prescrições Médicas na Atenção Primária em Saúde. Rev Uningá Review. 2018;29(1):54-60.
26. Teixeira CS, Fonseca A, Filardi R, De Araújo S, Mendonça M, Ribeiro MÂ. Avaliação da prática farmacêutica na perspectiva dos pacientes: uma revisão integrativa. Japhac. 2021;(7):53-78.

27. Soterio AK, Santos AM. A automedicação no Brasil e a importância do farmacêutico na orientação do uso racional de medicamentos de venda livre: uma revisão. Rev. Grad. 2016;9(2):1-15. 